\title{
Study on the Degradation Effect of Three Organophosphorus Hydrolase Mutant on Sarin
}

\author{
Qiamin $\mathrm{Gu}^{1,2, \mathrm{a}}$, Chuanxin $\mathrm{Zhao}^{2}$, Hailing $\mathrm{Xi}^{3, \mathrm{~b}}$, Min $\mathrm{Liu}^{2, \mathrm{c}}$, Gaoyun Chen ${ }^{2}$ \\ 166072 PLA troops, 100043, Beijing, China \\ ${ }^{2}$ Institute of NBC Defense, 102205, Beijing, China \\ ${ }^{3}$ State Key Laboratory of NBC prevention for Civilian, 102205, Beijing, China;
}

\begin{abstract}
Organophosphorus hydrolase can effectively degrade organic phosphorus compounds such as sarin. In this study, we constructed a recombinant Bacillus subtilis mutant expressing organophosphorus hydrolase, measured the effect of the mutant on the degradation rate of nerve agent sarin, and selected the optimal mutation scheme. Three different hydrolase mutant genes, 257L, 257Y and 303T, were ligated to PMA0911 vector and transferred into Bacillus subtilis WB800 to construct the target recombinant strain successfully. The recombinant bacteria secreted the target protein by fermentation. The effect of enzyme protein on the degradation of sarin was determined by the benzidine method. The optimal mutant was screened, and its enzymatic performance was explored. The effects of three organophosphorus hydrolase mutants on the hydrolysis rate of sarin were detected. The results showed that the $257 \mathrm{Y}$ mutant accelerated the hydrolysis of sarin significantly. Point mutation can improve the enzyme activity of wild-type organophosphorus hydrolase to a certain extent, laying the foundation for subsequent in-depth research.
\end{abstract}

\section{Introduction}

With the improvement of human life requirements and the increasing development of industrial production, many artificially synthesized pollutant compounds that are difficult to be rapidly degraded and transformed by natural microorganisms have entered the natural world. Among them, Organophosphate compounds (Ops), which are mass-produced and used on a large scale at home and abroad, have caused environmental pollution and damage to the ecological balance to a point that cannot be ignored. The most commonly used organophosphate compounds in people's lives are Organophosphorus Pesdcidcs. There are many varieties of organophosphorus pesticides, and there are more than 30 kinds used in my country alone, and they are all highly toxic. The excessive application of these pesticides pollutes the soil and water, and at the same time destroys the ecological balance of nature. At the same time, Organophosphate nerve agents (such as sarin and VX) with similar structures are highly toxic and lethal organophosphate compounds. These residual organophosphate compounds can inhibit the activity of acetylcholinesterase, cause the accumulation of acetylcholine, trigger a series of symptoms of neurotoxicity, and even cause death.

Sarin is a typical G-type neurotoxic agent. It has very weak fruit flavor, which is colorless and transparent liquid, easy to mix with water, and can be dissolved in organic solvent, easy to penetrate porous surface and painted surface. Of all the nerve palsy agents, sarin had

* Corresponding author: ${ }^{\mathrm{a}}$ gqm@nbcde.cn

bfhxihl@163.com, c1m@nbcde.con the highest volatilization. It has a strong contractive effect on human pupil. When sarin invades human body in various ways, its toxic effect is to damage nerve conduction and its latency is very short. There are three main aspects of sarin's effect on the body: one is to selectively inhibit cholinesterase activity, and make ACh accumulate in vivo, which causes the dysfunction of cholinergic nervous system; the second is that the poison acts on cholinergic receptor; third, the effect of the poison on the non cholinergic nervous system. The main symptoms are paralysis of respiratory function, mydriasis, severe pain of gastrointestinal spasm and secretion of body fluids. organophosphate compounds. These residual organophosphate compounds can inhibit the activity of acetylcholinesterase, cause the accumulation of acetylcholine, trigger a series of symptoms of neurotoxicity, and even cause death.

In order to protect human health and protect the ecological environment on which we depend, researchers have been looking for effective ways to eliminate pollution. The physical and chemical methods used in traditional methods to degrade organophosphate compounds usually have disadvantages such as high energy consumption, high cost, low efficiency, and secondary pollution that is not conducive to elimination; while organophosphorus hydrolase is a natural macromolecule For substances, enzymatic degradation has the advantages of mild reaction conditions, high efficiency, economic safety, thorough degradation, and no secondary pollution. It has become a hot issue that has been widely concerned and studied hard by all 
countries in the world. Among them, Organophosphorus hydrolase $(\mathrm{OPH})$ is a natural protease that can effectively hydrolyze organophosphate compounds.

$\mathrm{OPH}$ was originally isolated and purified from Flavobacterium sp. ATCC $27551^{[1]}$ and P. diminuta MG ${ }^{[2]}$, and is a typical phosphotriesterase. It hydrolyzes the phosphoric acid bond (P-O bond, P-CN bond, P-F bond and $\mathrm{P}-\mathrm{S}$ bond) between the phosphorus atom and the electrophilic atom to dissociate the group, thereby hydrolyzing many organophosphate triesters and thioesters ${ }^{[3]}$. Wild bacteria OPH has low activity in degrading organophosphorus pesticides or organophosphorus nerve agents and cannot meet the requirements for rapid disinfection. Therefore, it is one of the current research hotspots in biological disinfection technology to change the active site of organophosphorus hydrolase and improve the performance of the enzyme through protein engineering.

In order to improve the enzymatic performance of organophosphorus hydrolase and increase protein expression, this study linked the organophosphorus hydrolase gene after point mutation to the PMA0911 vector and transferred it to Bacillus subtilis for protein expression. The benzidine method was used to detect the degradation ability of the expressed protein to the organophosphate poison sarin, and the influence of different mutation sites on the enzyme activity was analyzed.

\section{Materials and Methods}

\subsection{Materials}

(1) Bacillus subtilis wb800, plasmid pma0911 and mutant particle of OPH were preserved in our laboratory.

(2) Main reagents: plasmid small extraction kit, gel Recovery Kit, ndei endonuclease, EcoRI endonuclease, T4 DNA ligase, DNA marker, protein marker, and the above reagents are all purchased from Tiangen biochemical technology (Beijing) Co., Ltd.

\subsection{Methods}

\subsubsection{Construction of plasmid of organophosphorus hydrolase}

A pair of primers was designed according to the sequence of organophosphorus hydrolase gene: upstream primer 5 '- ggaggattccattg-3' (NdeI); the downstream primer 5 '- gaattccgg-3' (EcoRI) was used to amplify the target gene containing restriction site from the $\mathrm{OPH}$ gene with different mutation sites stored in the laboratory. The target gene and plasmid pMA0911 were extracted by plasmid small extraction kit, respectively. After NdeI and EcoRI double enzyme digestion, agarose gel electrophoresis was performed. The target OPH gene fragment and pMA0911 fragment were recovered and purified by agarose gel Recovery Kit. The expression plasmid PMA OPH was constructed by linking the $\mathrm{OPH}$ gene with the vector pma0911 by T4 DNA ligase.

\subsubsection{Transformation of PMA OPH into Bacillus subtilis}

The text of your paper should be formatted as follows:

PMA OPH was transferred into competent E. coli, coated on LB solid medium containing ampicillin, and cultured overnight in $37{ }^{\circ} \mathrm{C}$ incubator. The resistant transformants were selected and the plasmids were extracted after expansion. B. The preparation and transformation of subtilis competent cells were carried out by spizizen method ${ }^{[4][5]}$. The transformants were coated on the selective plate containing kanamycin, and cultured overnight at $37^{\circ} \mathrm{C}$ and $180 \mathrm{r} / \mathrm{min}$ to obtain the target recombinant bacteria.

\subsubsection{Activity determination of recombinant organophosphorus hydrolase}

The recombinant strain was inoculated into $30 \mathrm{ml}$ TB expression medium and incubated at $37^{\circ} \mathrm{C}$ and $180 \mathrm{r} / \mathrm{min}$ for $24 \mathrm{~h}$. The supernatant was centrifuged at $4000 \mathrm{R} / \mathrm{min}$ for $10 \mathrm{~min}$. The concentration of $\mathrm{BCA}$ protein in supernatant was determined. The enzyme degradation ability of GB was detected by quantitative method of perbenzidine, and the hydrolysis ability of recombinant strain was tested.

Determination of BCA protein concentration: the BCA protein concentration determination kit of Solarbio company was used. Under alkaline condition, $\mathrm{Cu}^{2+}$ was reduced to $\mathrm{Cu}^{+}$by protein, and $\mathrm{Cu}+$ formed a purple blue complex with BCA reagent. The absorption value at $562 \mathrm{~nm}$ was determined and the protein concentration was calculated.

Drawing of sarin standard curve: weigh $100 \mu \mathrm{g} / \mathrm{ml}$ GB standard solution $0,50,100,150,200,250$, and add $0.01 \mathrm{M}$ PBS solution to $500 \mu 50$. Add $125 \mu \mathrm{L}$ acetone, $125 \mu \mathrm{L} \quad 0.5 \%$ benzidine solution and $500 \mu \mathrm{L} \quad 0.25 \%$ sodium perborate solution. The absorbance at $406 \mathrm{~nm}$ was measured.

Detection of enzyme activity by peroxybenzidine quantitative method: sodium perborate decomposes hydrogen peroxide in alkaline water, and hydrogen peroxide reacts with Class $G$ agents to form peroxyphosphonic acid. This peroxyphosphonic acid has strong oxidation ability and can oxidize benzidine to form colored azo fuel, and has a specific absorption peak at $406 \mathrm{~nm}$. The absorbance value of the sample at $406 \mathrm{~nm}$ is detected, The content of residual GB and enzyme activity were calculated.

The reaction system was $1.25 \mathrm{ml}$ : The crude enzyme solution was diluted with $0.01 \mathrm{M}$ PBS solution to make the protein concentration consistent. Take $250 \mu \mathrm{L}$ of diluted enzyme solution and add $250 \mu \mathrm{L}$ of $100 \mu \mathrm{g} / \mathrm{mL}$ sarin. After reacting at room temperature for 5 minutes, add $125 \mu \mathrm{L}$ of acetone, $125 \mu \mathrm{L}$ of $0.5 \%$ benzidine aqueous solution and $500 \mu \mathrm{L}$ of $0.25 \%$ sodium perborate aqueous solution. The content of residual GB and enzyme activity were calculated. The milligram of sarin per milligram of protease per minute was defined as the enzyme activity $\mathrm{U}$ of organophosphorus hydrolase. 


\section{Results}

\subsection{Construction of recombinant strain}

According to 2.2.1 and 2.2.2, the recombinant strain was constructed. The strain with h2571 mutation was numbered as No.1, the strain with $257 \mathrm{y}$ mutation was numbered as No.2, the strain with $303 \mathrm{t}$ mutation was numbered as No.3, and the strain with non mutant $\mathrm{OPH}$ was numbered as No.4. The results of double enzyme digestion after plasmid extraction were shown in Figure 1. The plasmid was digested by double enzymes and formed two bands, about $7000 \mathrm{bp}$ and $1000 \mathrm{bp}$, which corresponded to pma0911 vector and OPH gene fragment respectively. The results showed that the recombinant strain was wb800 Bacillus subtilis carrying PMA OPH.

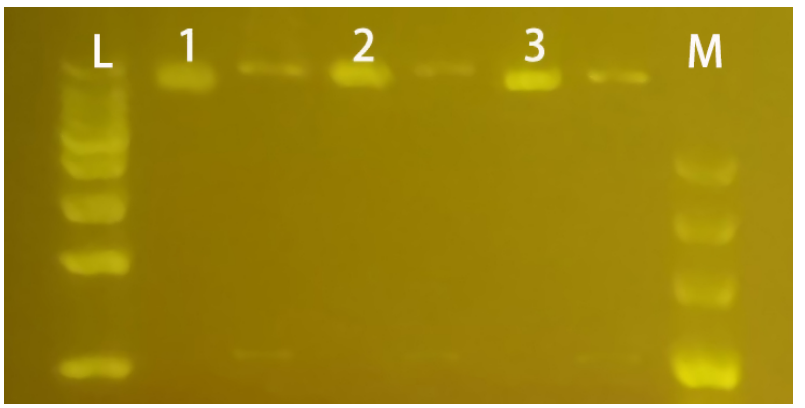

Figure 1. Double enzyme gel electrophoresis.

$\mathrm{L}$ is DNA leader; 1 is gene 1 , and the right band is double digestion of gene $1 ; 2$ is gene 2 , and the right side is double enzyme digestion of gene $2 ; 3$ is the No. 3 gene, and the right side is the No. 3 gene digested by double enzymes; $\mathrm{M}$ is DNA marker 3.

\subsection{Fermentation expression of recombinant bacteria}

After the recombinant strain was fermented and expressed in accordance with 2.2.3, the supernatant was taken for SDS-PAGE protein electrophoresis, and the results are shown in Figure 2. Compared with the original expression band of wb800 Bacillus subtilis, the recombinant strain carrying PMA OPH and the three mutants showed obvious protein bands at $36 \mathrm{kDa}$, and the recombinant strain successfully expressed high concentration of organophosphorus hydrolase. In the future, some methods will be used for extracellular expression to simplify the purification process.

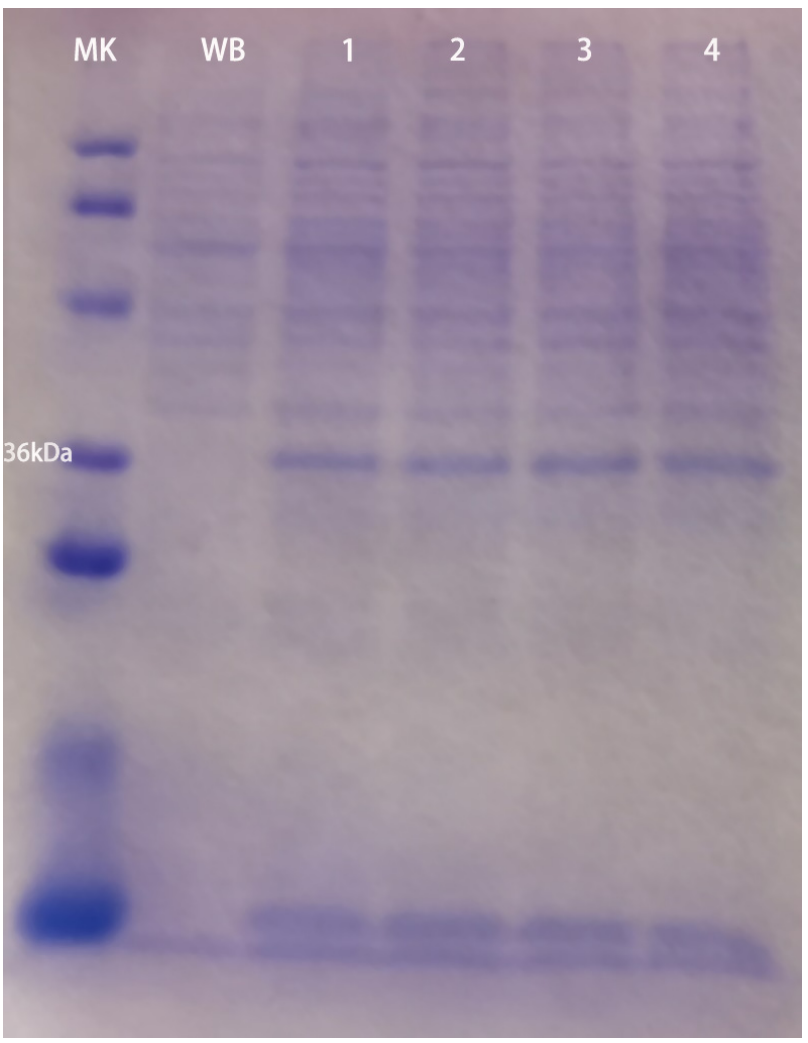

Figure 2. Gel electrophoresis of SDS protein MK is a protein marker; WB was Bacillus subtilis wb800; 1 is strain $1 ; 2$ is strain $2 ; 3$ is strain 3 .

\subsection{Establishment of sarin standard curve}

The standard curve of sarin was drawn according to 2.2.3.

Table 1. Sarin standard curve

\begin{tabular}{|c|c|c|c|c|c|c|}
\hline & 1 & 2 & 3 & 4 & 5 & 6 \\
\hline $\begin{array}{c}\text { Sarin dosage } \\
(\mathrm{mL})\end{array}$ & 0 & 0.05 & 0.1 & 0.15 & 0.2 & 0.25 \\
\hline $\begin{array}{c}\text { Sarin standard } \\
\text { concentration } \\
(\mu \mathrm{g} / \mathrm{mL})\end{array}$ & 0 & 20 & 40 & 60 & 80 & 100 \\
\hline $\mathrm{A} 406$ & 0.052 & 0.130 & 0.190 & 0.255 & 0.319 & 0.386 \\
\hline
\end{tabular}

\section{Sarin standard curve}

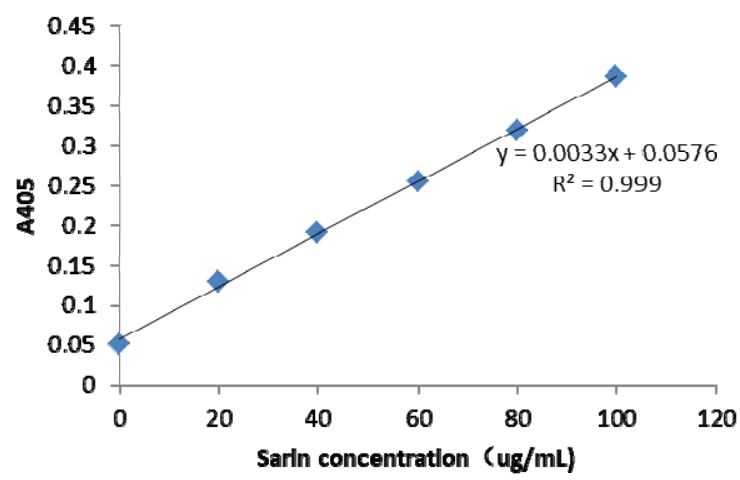

Figure 3. Sarin standard curve 


\subsection{Determination of hydrolase activity}

According to 2.2.3, the enzyme activity of protein secreted by each recombinant strain was tested. The experimental results are shown in Table 2. Compared with the wild-type $\mathrm{OPH}$ without mutation, h2571 and h257y increased the enzyme activity u by $18 \%$ and $32 \%$ respectively, that is, the hydrolysis rate of sarin increased, while $1303 t$ decreased the enzyme activity $u$, that is, the hydrolysis rate of sarin decreased.

Table 2. Enzymatic activity of hydrolysis of sarin by $\mathrm{OPH}$ mutant

\begin{tabular}{|c|c|c|c|c|c|}
\hline & CG & WT & H257L & H257Y & L303T \\
\hline $\begin{array}{c}\text { degradation } \\
\text { rate of sarin } \\
(\%)\end{array}$ & 0.60 & 40.68 & 47.92 & 53.64 & 29.34 \\
\hline U & - & 0.362 & 0.426 & 0.477 & 0.261 \\
\hline $\begin{array}{c}\text { Enzyme } \\
\text { activity } \\
\text { improvement }\end{array}$ & - & 1 & $18 \%$ & $32 \%$ & $-28 \%$ \\
\hline
\end{tabular}

\section{Discussion}

In this study, the expression and enzyme activity of three OPH mutants in Bacillus subtilis were successfully determined. Compared with E.coli, the expression performance, safety and operability of Bacillus subtilis were improved. This study verified that the hydrolysis effect of organophosphorus hydrolase on sarin can be changed by controlling the amino acid sequence of some key sites. In this study, we selected two mutation sites, 257 site in the large hydrophobic pocket (H254, H257 and L271) of the active center of OPH. By analyzing the protein structure, we mutated histidine with positive charge and hydrophilic polarity to tyrosine with no charge and hydrophobic polarity, which may change the charge of the hydrophobic pocket and enhance the hydrophobicity of the hydrophobic pocket, The interaction between the pocket structure and the substituent of the substrate molecule was promoted. The 303 site was located in a small hydrophobic pocket (M317, G60, I106, L303 and S308). After the nonpolar leucine at 303 site was mutated into the polar threonine, the hydrolysis ability of the hydrolase was inhibited.

\section{Conclusions}

In this experiment, we successfully constructed three mutants of recombinant Bacillus subtilis expressing organophosphorus hydrolase, respectively measured the effect of the mutants on the degradation rate of nerve agent sarin, and screened out the optimal mutant h257y, which significantly improved the enzyme activity of sarin degradation, and the enzyme properties of the mutants were relatively stable. In this experiment, only three mutation sites were selected for discussion, which verified that changing the amino acids in the enzyme activity center site of OPH can effectively change its enzyme activity. In the follow-up research, we can combine the computer software fitting function to analyze the spatial structure of different sites, different amino acids, binding sites and various forces, combined with the hydrolysis principle of organophosphorus hydrolase, to find more key sites and better mutation direction.

At the same time, with the development of degrading enzyme research, many degrading strains with high enzyme activity have been successfully developed, but they are still in the stage of laboratory research. There are still many problems to be solved when these new degradation methods are transferred from laboratory to practical application. Two methods can be considered for the practical application of recombinant strains. One is to quickly resuscitate the strains before use and to secrete and express them. When the protein secretion concentration reaches a certain level, it can be used for the biological decontamination of organophosphorus substances and nerve agents. The other is to directly put recombinant bacteria in the process of environmental decontamination, which is expected to produce organophosphorus hydrolase in the process of natural growth and passage, and participate in the degradation of organophosphorus substances, so as to achieve the purpose of ecological decontamination.

It can be predicted that with the discovery of new degrading microorganisms, the emergence of degrading enzymes and the development of bioengineering technology, biodegradation technology will play an increasingly important role.

\section{References}

1. Dave K I, Miller C E, Wild J R. (1993). Characterization of organophosphorus hydrolases and the genetic manipulation of the phosphotriesterase from Pseudomonas diminuta[J]. Chemico-Biological Interactions, 87(1-3):55-68.

2. Pogell B M, Rowland S S, Steinmann K E, et al. (1991). Genetic and biochemical evidence for the lack of significant hydrolysis of soman by a Flavobacterium parathion hydrolase.[J]. Applied and Environmental Microbiology, 57(2):610-611.

3. Casey, M, TheriotAmy, et al. (2011). Hydrolysis of organophosphorus compounds by microbial enzymes[J]. Applied Microbiology\&Biotechnology, , 89(1):35-43.

4. C.A. Anagnostopoulos, Spizizen J. (1961). Requirements for transformation in bacillus subtilis[J]. Journal of Bacteriology, 81(5):741.

5. Spizizen, J. (1958). Transformation of biochemically deficient strains of bacillus subtilis by deoxyribonucleate[J]. Proceedings of the National Academy of Sciences of the United States of America, 44(10):1072-1078. 